\title{
Cervical Cancer - Bench to Bedside
}

\author{
T. Rajkumar \\ Prof. and Head, Dept. of Molecular Oncology, \\ Cancer Institute (WIA), Adyar, Chennai.
}

\begin{abstract}
Cervical cancer is the second most common cancer in Indian women and 4th most common cancer in women world-wide. Over nearly two decades, we have carried out epidemiological and molecular studies in cervical cancer, with an intent to identify potential early diagnostic biomarkers, predictive and prognostic markers, develop newer therapies against cervical cancer and identify potential new targets for therapy.

Our studies had identified 14 high risk and 10 low risk human papilloma virus (HPV) in our cervical cancer patients for the first time; had identified life style related cofactors in the development of cervical cancer (paan chewing, parity, early age at first sexual intercourse and first childbirth, husband with two or more sexual partners). We have developed a p16 ELISA kit for cervical cancer screening for use at point of care like PHC's; identified a 7 gene signature which help identify patients who can be treated with radiotherapy alone; identified potential prognostic markers for use in the clinic; developed the country's first Dendritic cell vaccine therapy for cervical cancer and completed the phase 1 study; have identified newer potential therapeutic targets for treatment of cervical cancer.
\end{abstract}

Keywords: Cervical cancer; Human papilloma virus; p16 ELISA; Predictive and Prognostic markers; UBE2C; Dendritic Cell Vaccine for treatment.

Correspondence : Dr. T. Rajkumar, Prof. and Head, Dept. of Molecular Oncology, Cancer Institute(WIA), Adyar, Chennai-600020, Email: drtrajkumar@gmail.com.

DR. R.V. RAJAM ORATION delivered during NAMSCON 2015 at the All India Institute of Medical Sciences, Patna. 


\section{INTRODUCTION :}

Cervical cancer is the 2nd most common cancer in Indian women and 4th most common world-wide (1). High risk human papilloma viruses (HPV) are considered to be important in the genesis of these cancers (2). However, most of the women infected $(>95 \%)$ will clear the infection but a Few can have HPV induced pre-cancerous changes which can worsen to invasive cancers, indicating that other factors are also important in the genesis of the cancer. Identifying additional life style related factors which increase or decrease the risk can help better control the disease development.

Cervical cancer is a disease of relatively slow progression, going through different grades of dysplasia before becoming invasive (3). The standard screening procedure for cervical cancer is the Pap smear. However, in a country as large as India and with a huge population load, it is near impossible to use PAP smear for screening, simply because we do not have enough Pathologists/Cytologists. Low cost techniques like Visual inspection after application of acetic acid (VIA) or Lugol's iodine (VILI) have lower sensitivity and specificity. HPV testing has the drawback of needing re-testing after a year to confirm persistence of infection, as a single test cannot confirm if it is a transient infection. There is, therefore, an urgent need for a simple, low cost and objective testing to identify the pre-cancerous lesions of the cervix at the Primary Health Centres itself.
The standard line of management of stage IIB and stage IIIB till the end of the 20th century was radical radiotherapy. The cure rates with radical radiotherapy was around $60 \%$ for stage IIB and around $40 \%$ in stage IIIB. The introduction of cisplatin based concurrent chemoradiotherapy has helped improving the cure rates by nearly $10 \%$. The question is, can we identify those patients who could be cured with the radical radiotherapy itself thereby avoiding the cost and toxicity of the chemotherapy.

In view of the stagnation of the cure rates newer approaches and newer therapeutic agents need to be identified. One promising approach is to use therapeutic immune based approaches such as Dendritic cell vaccine therapy in the treatment of cervical cancer. Additionally identification of newer therapeutic targets and development of drugs to those targets is essential to improve the cure rates in cervical cancer.

\section{A. Epidemiological studies in cervical cancer :}

International Agency for Research on Cancer (IARC, Lyon) funded the case control study on HPV in cervical cancer conducted from June 1998 to May 1999 in the Cancer Institute (WIA) (4). Inclusion criteria for case subjects were: (i) histologic confirmation of invasive cervical cancer (ICC) diagnosis; (ii) no previous cancer treatment; (iii) lack of physical or mental impairments that would have made the interview impossible; (iv) willingness to provide 
informed consent. Healthy control women were either other patients in the Institute or visitors of patients other than women with cervical cancer and were agematched to ICC cases within 5-year age groups. Exclusion criteria for control women included: (i) a diagnosis of anogenital tract cancers (i.e., cervix, vagina, vulva, anal canal), cancer of the breast, endometrium, ovary or colon, benign genital tumours and tobacco-related diseases (e.g., coronary heart disease, chronic bronchitis and cancer of the head and neck, lung and bladder); (ii) a history of hysterectomy or cervical conization; (iii) physical or mental problems and (iv) not willing to provide informed consent.

All the cases $(n=205)$ and control $(n=213)$ women underwent a gynaecologic examination and ectocervical \& endocervical cell collection was done using appropriate spatulas and brushes. The cells were stored at $-70^{\circ} \mathrm{C}$ and then shipped to IARC, Lyon. HPV detection was done using PCR followed by an Enzyme immunoassay containing probes which would detect high risk and low risk HPV subtypes.

After excluding inadequate samples, we had 191 ICC and 184 control women's samples with HPV data. High risk HPV was detected in 190/191 cases (178/179 squamous cell carcinoma; $12 / 12$ adenocarcinoma) and in 40 of the 184 control women. Low risk HPV only was seen in 11 of the control women but not in ICC cases. HPV infection with any subtype was associated 498-fold increased ICC risk. HPV16 was the most common subtype seen in cases and controls, followed by HPV18 and 33 . Multiple subtype infection was seen in $17 \%$ of cases and $4 \%$ of controls.

This was the first study from the country to look at all the HR and LR HPV subtypes. It confirmed the strong association of HR-HPV subtypes with ICC. It also for the first time showed the incidence of HR-HPV infection in control women to be as high as $22 \%$. The study also identified several risk factors including paan chewing with and without tobacco (OR 2.3) with a dose dependent effect; parity; women with husbands who had extramarital affairs; age at first intercourse $<15$ years compared to $\geq 21$ years was associated with OR 2.2; age at first child birth $(<17$ years compared to $\geq 19$ years was associated with OR 2.2); more than one sexual partner; all of which increased the risk. High vegetable and fruits intake was associated with a decreased risk for ICC (OR 0.5) (5). The data from the study was included in an International Collaboration of Epidemiological studies of cervical cancer co-ordinated by IARC, Lyon. This reanalysis of data involving 25 epidemiological studies with more than 16000 ICC and more than 30,000 controls showed:

\section{Reproductive factors (6) :}

a. The risk for developing invasive cervical cancer increases with parity ( $\geq 7$ full term pregnancy compared with $1-2)$.

b. Early age at first full term pregnancy 
( $<17$ years versus $\geq 25$ years) was associated with increased risk for invasive cervical cancer and for CIN3/CIS lesions.

\section{Tobacco smoking (7) :}

a. Tobacco smoking (current smokers and past smokers) had an increased risk for squamous cell carcinoma (SCC) of cervix. The RR in current smokers was 1.6 (95\% CI: $1.48-$ $1.73 ; \mathrm{p}<0.001)$ and in past smokers was 1.12 (95\%CI: $1.01-1.25)$.

b. The risk in current smokers for the development of SCC of the cervix, increased with increasing number of cigarettes smoked and on the younger age at which they started smoking $(\mathrm{p}<0.001)$.

c. Tobacco smoking was not associated with an increased risk for Adenocarcinoma of the cervix.

\section{Hormonal contraceptives (8):}

a. Individuals using combined oral contraceptives for 5 or more years had a RR of 1.9 (95\% CI: $1.69-$ 2.13) for the development of invasive cervical cancer.

b. A similar risk was also seen for CIN3/CIS.

c. Injectable progestagen-only contraceptives showed a slightly raised risk for invasive cervical cancer, when used for 5 or more years (RR 1.22 [95\% CI: 1.01 $1.46] \mathrm{p}=0.03)$.

d. The risk of cervical cancer declines once the drugs are stopped and by 10 years or more, the risk is similar to that of a never user.

\section{Sexual behaviour (9) :}

a. A lady with many sexual partners $(\geq 6)$ has more than two fold risk for development of invasive cervical cancer and for CIN3/CIS, after conditioning on age, study and age at first intercourse.

b. The age at first intercourse is also a risk determinant, with $\leq 14$ years of age at first intercourse having a greater than 2 fold risk, when compared with age at first intercourse of $\geq 25$, and after conditioning for age, study, number of sexual partners and reproductive factors.

\section{Education (10) :}

a. Low education level was found to be associated with cervical cancer but not with HPV infection.

\section{B. Molecular studies in cervical cancer:}

Microarray studies were carried out on normal cervical tissues $(\mathrm{n}=5)$, different grades of dysplasia (CIN1 \& 2 $n=4 ; \operatorname{CIN} 3 / \operatorname{CIS} n=4)$ and ICC $(n=28)$. Differentially expressed genes were then validated using quantitative Real Time PCR (qRT-PCR). Some of the genes were then validated at the protein level using Immunohistochemistry (IHC) (11). 


\section{1. p16ELISA :}

Our qRT-PCR data identified p16 as being highly expressed in high grade CIN3/CIS and in ICC compared to normal and low grade CIN1/CIN2. IHC showed the protein to be overexpressed in tumours and in high grade CINs. We then proceeded to develop a sandwich ELISA for detection of p16 using commercially available antibodies. Concurrently we started raising monoclonal antibodies to p16 using recombinant $\mathrm{p} 16$ protein which we had expressed and purified from bacterial cells.

The Sandwich ELISA using commercial antibodies was validated using recombinant $\mathrm{p} 16$. Once we had the monoclonal antibodies raised and characterized by us, we used a combination of the monoclonal antibodies in the sandwich ELISA and were able to detect reliably $1 \mathrm{pg}$. We then evaluated the ELISA using HeLa cells, and were able to detect upto 500 cells reliably. One of the problem we faced was assessing the adequacy of the cervical scrape sample. In PAP smear, the Pathologists can assess whether the sample is adequate or not for interpreting and can order for a fresh sample if found inadequate. In an ELISA, this was not possible and hence we developed and added another ELISA for cytokeratin level. Cytokeratin is expressed in almost all epithelial cells and an antibody against pan-cytokeratin can identify the epithelial content. By running the p16 and cytokeratin ELISA in parallel, we were able to show the adequacy of the sample and also determine the levels of p16 expression. This was a major advance over an existing ELISA kit for p16 developed and marketed in Germany. We have done the preliminary assessment of the functioning of the ELISA using well annotated clinical samples from Nargis Dutt Memorial Cancer Hospital, Barshi, wherein a cervical cancer screening program has been going on for more than a decade. Patent has been applied for the p 16 kit [Application number 475/CHE/2014, filed on 03-02-2014]. Further validation is being planned by using blinded samples from Barshi which will be followed by independent validation in 3 major centres in India involving at least 1000 samples. We are in the process of transferring the Technology to a Govt. of India subsidiary for commercialization of the kit.

\section{Predictive markers for radiation response in cervical cancer :}

As mentioned earlier, the addition of concurrent chemotherapy with radical radiation improves the cure rate by around $10 \%$. However, we do not have a means of identifying who would fail treatment, so that chemotherapy could be reserved for them thereby minimizing toxicity and cost of the chemotherapy. To this end we had carried out gene expression studies using microarray as described in (12) which was then validated using qRT-PCR.

Using 24 samples from patients who had undergone therapy with more than 3 years follow-up, we developed a scoring method based on the qRT-PCR data. The cut-off was set at $40 \%$ of the potential total 
T. Rajkumar 149

score possible. A 7 gene signature score was found to identify patients likely to fail treatment. At the cut-off score set, all the failed samples were identified as high risk. The sensitivity, specificity, PPV and NPV were $0.64,1.0,1.0,0.67$, respectively.

This needs further validation using a larger sample size, in samples collected from patients who had undergone radical radiotherapy only. We are hoping to collaborate with other centres which would have frozen tumour samples available of patients treated with radiotherapy only.

\section{Prognostic markers for cervical cancer :}

At the Institute, most of the cervical cancer cases are stage IIB and IIIB (nearly $80 \%$ ) and of high grade ( $80 \%)$. Since stage and grade of the tumours are strong prognostic indicators, this meant that most of our patients were high risk group and additional markers are required to better sub-stratify them.

Our department had evaluated several proteins for their potential as prognostic markers, overexpression of BCL2 was found to be associated with poor response to treatment with Radical radiotherapy and more patients failed treatment after achieving a complete response, as well (Table 1 - BCL2). This was independent of the stage of disease (13). C-myc oncoprotein overexpression was also likewise associated with poor prognosis independent of the stage (14).

\section{Identification of molecular targets in cervical cancer :}

Based on our gene expression studies (microarray and qRT-PCR) we identified several potential targets for treatment in cervical cancer, including UBE2C, MELK, ISG15, STAT1, MMP3, MMP1 (11).

UBE2C which is a Ubiquitin conjugating enzyme E2C, is expressed at high levels in cervical tumours and high grade dysplasia but at low levels or is absent in normal tissues and low grade dysplasia. Using a functionally inactivated dominant-negative mutant of UBE2C (C114S), we showed that the mutant protein alters the tumorigenicity (soft agar cloning assay) and sensitises cancer cells (cervical cancer cell lines) to radiation (Clonogenic assay) (15).

We are now involved in in-silico modelling and docking of compounds to the active site including C114. Compounds showing UBE2C blocking activity will be taken forward for further evaluation.

\section{Development of dendritic cell vaccine therapy for cervical cancer :}

Immune perturbation is one of the critical factor in the development of cancer. Every day in a normal individual there are millions of cells undergoing proliferation which is essential to maintain normal physiological functions. Some of the cells undergoing proliferation will have error in DNA sequence. The first 
line of defence in this is the DNA repair mechanism which can correct the errors and if the cell fails to correct the error and tries to enter another cell division, apoptosis is triggered, so that the abnormal cell does not result in clonal proliferation. In addition, several environmental and dietary factors can damage the cellular DNA and again the DNA repair mechanisms ensure the DNA integrity is maintained. However, in spite of well- regulated DNA repair process, sometimes the error can be propagated to daughter cells. At this level, the immune system comes into play with its continuous surveillance, detecting these abnormal cells and destroying them. In some situations, the immune system is also compromised and the developing tumour cells can alter its MHC protein expression and secrete factors which can result in tolerance to the developing tumour. There are several ways to break the immune tolerance, one of which is to use primed dendritic cells to overcome the resistance.

Dendritic cells (DC) are the most potent antigen presenting cells and can help initiate a strong immune response. In the tumour microenvironment, the dendritic cells are either inactive or can be inhibitory to the immune response due to tumour / stroma derived factors such as IDO (Indoleamine deoxygenase). We and others have shown that DCs can be generated from peripheral blood derived monocytes and these can then be matured into primed dendritic cells and then injected back into the patients to generate a robust immune response.

\section{Generation and characterization of mature primed Dendritic cells for DC vaccine :}

Under funding from Dept. of Biotechnology, Government of India, we first standardized the protocol for generation of DC's and then functionally characterized them (16). Briefly, we obtained peripheral blood mononuclear cells from patients and then plated them in plastic petridishes. Monocytes adhere to the plate while other cells float up and are removed and used for functional assays. The monocytes are then grown in the presence of IL4 and GM-CSF (100ng/ml each) in therapeutic grade serum free AIM-V medium for 7 days. The floating immature DCs were transferred on the 7 th day to fresh plate containing AIM-V medium and then exposed to tumour antigens derived either from patient's own tumour lysate or mRNA from the tumour for 4 hours. IL- $1 \beta$ and TNF- $\alpha$ are added at $100 \mathrm{ng} / \mathrm{ml}$ and incubated for another 3 days. The mature primed cells were then characterized using FACS and functional assays.

Mature primed DC's were HLA-DP, DQ, DR +++; CD86++; CD14 negative. In contrast, immature DC's were HLADP, DQ, DR +++; CD86+; CD14 negative, indicating that they are less likely to be good antigen presenter due to lower levels of co-stimulatory molecule CD86. The tumour lysate primed DC's were more effective in functional assays compared to mRNA primed DC's particularly when primed in a ratio of 3 tumour cell : 1 DC. 
2. A Phase 1 clinical trial to evaluate the toxicity of $D C$ vaccine in recurrent and/or metastatic HPV positive cervical cancers :

This was a part of the DBT funded study. The study was a 3 arm study recruiting cervical cancer patients who had relapsed or had progressive disease after initial radical radiotherapy. Arm 1 patients received saline; Arm 2 received mature unprimed DC's; Arm 3 patients received mature tumour lysate (patient's own tumour) primed DC's. The inclusion and exclusion criteria have been published (16). Arm 1 was dropped after recruitment of 9 patients, as the DBT review committee found a satisfactory difference between patients receiving the DC's (primed or unprimed) compared to those receiving saline. 5 additional patients were recruited ( 3 in Arm 2 and 2 in Arm 3). Patients were given 3 doses every 14 days, of the appropriate DC vaccine at a maximum dose of $1 \times 10^{6}$ mature DCs per injection.

The DC vaccine was well tolerated with most of the patients having no side effects. Itching at the site of injection (1 patient), grade 1 fever possibly due to urinary tract infection (2 patients) and one patient had elevated serum Alkaline phosphatase. The Delayed Type Hypersensitivity (DTH) response was evaluated in 11 of the 14 patients recruited, with 2/4 Arm 2 and 2/4 Arm 3 patients tested being positive. In one of the Arm 3 patient who had locally persistent residual disease and a solitary lung
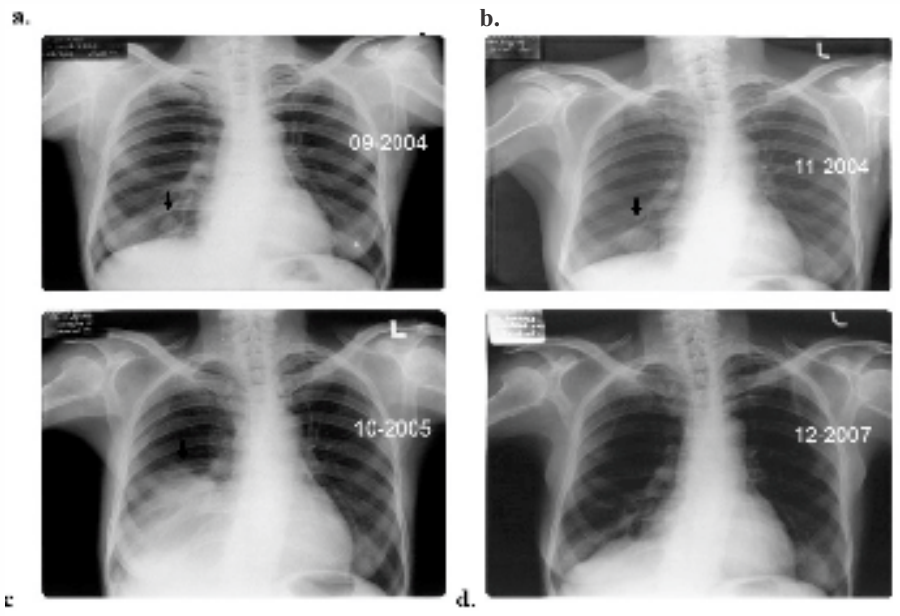

Fig. 1: Progress in the metastatic lung lesion in one of the patient.

Fig. 1a. Lung metastatic lesion detected at patient enrolment (indicated by

black arrow). Fig. 1b and $\mathrm{c}$ show progression of lesion after vaccination (1c. Was taken a year after vaccination). Fig. 1d. Shows complete regression of lesion three years after vaccination and 1.5 years after cisplatin chemotherapy. The patient is disease free 9 years after the chemotherapy in 2006. 
metastasis after radical radiotherapy, her local disease resolved within 6 months after her last dose. Her lung lesion progressed and she was advised symptomatic treatment only at her last follow-up in 2005 at which time the lesion was pushing her chest wall. In December 2007, when we were updating our followup information telephonically, she was found to be alive and well. She was then reviewed at the Institute and was found to be free of disease locally and at the metastatic site (Fig.1). She continues to be free of disease 9 years since receiving cisplatin. While cisplatin is known to produce complete response in metastatic sites, the disease relapses within 18 months to 2 years. That this patient is free of disease for more than 9 years suggests that the DC vaccine could have contributed to the sustained disease free status by triggering the memory T-cells when the tumour antigens were released due to the chemotherapy administered. The same patient had the strongest proliferative response as well as a strong DTH response, indicating that her immune system had been stimulated well.

Based on our data, we have been now funded by the Department of Science and Technology, Government of India for conducting a Phase 2 study. This will be a 3 arm study in untreated stage IIIB cervical cancer patients, with Arm 1 patients receiving standard therapy of concurrent chemo-radiation; Arm 2 patients receiving standard therapy of concurrent chemo-radiation plus DC vaccine primed with patient's own tumour lysate; Arm 3 patients receiving standard therapy of concurrent chemo-radiation plus DC vaccine primed with SPAG9 recombinant protein (developed by Prof. Anil Suri, National Institute of Immunology). DCGI clearance was obtained in Feb 2015 and hope to start the study by August 2015 .

\section{Conclusion :}

Work summarises our research in cervical cancer covering almost all aspects of the disease. There are several firsts in the country highlighted here - the first Epidemiological study to identify 14 HR HPV and 10 LR HPV subtypes in cervical cancer patients and healthy controls; first to identify a predictive marker for identifying patients who can be treated with radical radiotherapy alone; first to develop and conduct a phase 1 Dendritic cell vaccine clinical trial; first to develop a low cost ELISA for cervical cancer screening. Ours is an on-going endeavour to help control the disease.

\section{Acknowledgements :}

1. Dept. of Science and Technology

2. Dept. of Biotechnology

3. International Agency for Research on Cancer, Lyon, France

4. Dr. S. Krishnamurthi, Cancer Institute [WIA]

5. Dr. V. Shanta, Cancer Institute [WIA]

6. Dr. R. Sankarnarayanan, IARC

7. Dr. Silvia Franceschi, IARC

8. Dr. G. Selvaluxmi, Cancer Institute [WIA]

9. Dr. S. Shirley, Cancer Institute 
[WIA]

10. Dr. A. Sharmila, Cancer Institute [WIA]

11. Dr. G. Gopal, Cancer Institute [WIA]

12. Dr. R. Priya, Cancer Institute [WIA]

13. Dr. K. Sabitha, Cancer Institute [WIA]

14. Dr. B. MayilVahanan, Cancer Institute [WIA]

15. Dr. B.M. Nene, Nargis Dutt Memorial Cancer Hospital, Barshi

16. Dr. Sylla Malvi, Nargis Dutt Memorial Cancer Hospital, Barshi

\section{REFERENCES :}

1. Ferlay J, Soerjomataram I, Ervik M, et al. (2013). GLOBOCAN 2012 v1.0, Cancer Incidence and Mortality Worldwide: IARC Cancer Base No. 11 (Internet). Available from: http://globocan.iarc.fr.

2. Z Z r H a u se n H ( $\left.\begin{array}{llll}2 & 0 & 0 & 9\end{array}\right)$. Papillomaviruses in the causation of human cancer - a brief historical account. Virology 384: 260-265.

3. Arends MJ, Buckley CH, Wells M (1998). Aetiology, pathogenesis and pathology of cervical neoplasia. $J$ Clin Pathol 51: 96-103.

4. Franceschi S, Rajkumar T, Vaccarella S, et al. (2003). Human papilloma virus and risk factors for cervical cancer in Chennai, India: A case control study. Int J Cancer 107: 127-133.
5. Rajkumar T, Franceschi S, Vaccarella S, et al. (2003). The role of paan chewing and dietary habits in cervical carcinoma in Chennai, India. Br J Cancer 88: 1388-1393.

6. International Collaboration of Epidemiological Studies of Cervical cancer (2006). Cervical carcinoma and reproductive factors: collaborative reanalysis of individual data on 16,563 women with cervical carcinoma and 33,542 women without cervical carcinoma from 25 epidemiological studies. Int JCancer 119:1108-1124.

7. International Collaboration of Epidemiological Studies of Cervical cancer (2006). Carcinoma of the cervix and tobacco smoking: Collaborative reanalysis of individual data on 13,541 women with carcinoma of the cervix and 23,017 women without carcinoma of the cervix from 23 epidemiological studies. Int $J$ Cancer 118: 1481-1495.

8. International Collaboration of Epidemiological Studies of Cervical Cancer (2007). Cervical c a n c e r and hormon a 1 contraceptives: collaborative reanalysis of individual data on 16,573 women with cervical cancer and 35,509 women without cervical cancer from 24 epidemiological studies. Lancet 370: 1609-1621. 
154 Cervical Cancer - Bench to Bedside

9. International Collaboration of Epidemiological Studies of Cervical Cancer (2009). Cervical carcinoma and sexual behaviour: collaborative reanalysis of individual data on 15,461 women with cervical carcinoma and 29,164 women without cervical carcinoma from 21 epidemiological studies. Cancer Epidemiology Biomarkers and Prevention 18: 1060-1069.

10. Franceschi S, Plummer M, Clifford $\mathrm{G}$, et al. (2009). Differences in the risk of cervical cancer and human papillomavirus infection by education level. $\mathrm{Br} J$ Cancer 101:865-870.

11. Rajkumar T, Sabitha K, Vijayalakshmi N, et al. (2011). Identification and validation of genes involved in cervical tumourigenesis. BMC Cancer 11:80.

12. Rajkumar T, Vijayalakshmi N, Sabitha K, et al. (2009). A 7 gene expression score predicts for radiation response in cancer cervix. BMC Cancer 9:365.
13. Rajkumar T, Sharmila R, Majhi U, Selvaluxmi G, Vasanthan A, Shanta V (1998). Prognostic significance of $\mathrm{Bcl}-2$ and $\mathrm{p} 53$ protein expression in stage IIB and IIIB squamous cell carcinoma of the cervix. Eur $J$ Gynecol Oncol 19: 556-560.

14. Vijayalakshmi N, Selvaluxmi G, Mahji U, Rajkumar T (2002). cMyc oncoprotein expression and prognosis in patients with carcinoma cervix - An immunohistochemical study. Eur $J$ Gynecol Oncol 23: 135-138.

15. B ose MV, Gopisetty G, Ganeshrajah S, Rajkumar T (2012). Dominant negative Ubiquitin conjugating enzyme E2C sensitizes cervical cancer cells to radiation. Int J Rad Biol 88: 629-634.

16. Ramanathan P, Ganeshrajah S, Raghanvan RK, Singh SS, Rajkumar T (2014). Development and clinical evaluation of dendritic cell vaccines for HPV related cervical cancer-a feasibility study. Asian Pacific J Cancer Prev 15: 5909-5916. 